Check for updates

Cite this: RSC Adv., 2019, 9, 35356

Received 22nd September 2019 Accepted 17th October 2019

DOI: $10.1039 / c 9 r a 07687 e$

rsc.li/rsc-advances

\section{Ultra-performance liquid chromatography/mass spectrometry technology and high-throughput metabolomics for deciphering the preventive mechanism of mirabilite on colorectal cancer via the modulation of complex metabolic networks $\uparrow$}

\author{
Hui Sun, ${ }^{a} \mathrm{Ai}-$ hua Zhang, ${ }^{\mathrm{a}}$ Hong-lian Zhang, ${ }^{\mathrm{a}}$ Xiao-hang Zhou, ${ }^{\mathrm{a}}$ Xiang-qian Wang, ${ }^{\mathrm{a}}$ \\ Liang Liu ${ }^{b}$ and Xi-jun Wang (D) *abc
}

\begin{abstract}
Colorectal cancer (CRC) is a highly virulent and malignant disease and always accompanied by metabolic disorders. Currently, there are no effective therapeutic drugs for the treatment of CRC. High-throughput metabolomics approaches have been used to unveil the metabolic pathways related to several diseases. In this study, ultra-performance liquid chromatography/mass spectrometry-based high-throughput metabolomics was used for deciphering the potential preventive mechanism of mirabilite on CRC via the modulation of the associated metabolic disorders; a total of 28 differential biomarkers, including indole acetaldehyde, 5-hydroxyindoleacetic acid, hypoxanthine, retinal, retinal ester, linoleic acid, stearic acid, 6-deoxocastasterone, 2-hydroxybutyric acid and LysoPC, were identified in the APC min/+ mice. These differential biomarkers are involved in the tryptophan metabolism, glycerophospholipid metabolism and biosynthesis of unsaturated fatty acids. Note that these biomarkers and their disturbed metabolic pathways were also regulated by mirabilite. It has been found that the prevention of CRC by mirabilite is mainly associated with tryptophan metabolism; this study shows that high-throughput metabolomics can reveal the perturbed metabolic disorders targeted in the action mechanism of drug treatment.
\end{abstract}

\section{Introduction}

Colorectal cancer (CRC) is always detected in the middle and late stages due to which the mortality rate is very high; ${ }^{1}$ the 5 year relative survival rate is more than $90 \%$ when CRC is diagnosed in Phase I, and it decreases to $3 / 4$ in Phase IV. ${ }^{2-5}$ Therefore, early diagnosis of colorectal cancer is particularly important, which can significantly improve the efficacy of the treatment and the chances of survival. ${ }^{6}$ Currently, there are no effective therapeutic drugs for CRC treatment. Traditional Chinese medicine (TCM) has been considered as an abundant treasure house for disease treatment. Mirabilite, one of the TCM mineral agents, has a purgative action. ${ }^{7}$

${ }^{a}$ National Chinmedomics Research Center, Sino-America Chinmedomics Technology
Collaboration Center, National TCM Key Laboratory of Serum Pharmacochemistry,
Laboratory of Metabolomics, Department of Pharmaceutical Analysis, Heilongjiang
University of Chinese Medicine, Heping Road 24, Harbin, China. E-mail:
xijunwangls@126.com; Fax: $+86-451-82110818 ;$ Tel: +86-451-82110818
${ }^{b}$ State Key Laboratory of Quality Research in Chinese Medicine, Macau University of
Science and Technology, Avenida Wai Long, Taipa, Macau, China
${ }^{c}$ National Engineering Laboratory for the Development of Southwestern Endangered
Medicinal Materials, Guangxi Botanical Garden of Medicinal Plant, Nanning,
Guangxi, China

$\dagger$ Electronic supplementary information (ESI) available. See DOI: $10.1039 / \mathrm{c} 9 \mathrm{ra} 07687 \mathrm{e}$
It has been reported that mirabilite can be used in the treatment of gastrointestinal diseases such as acute peritonitis, pancreatitis and abdominal pain, ${ }^{\mathbf{8} 9}$ and it is suggested that mirabilite has a regulatory effect on intestinal diseases.

Metabolomics is a high-throughput tool to explore metabolites and unlock biological mysteries by detecting small molecule metabolites. ${ }^{10-15}$ As is known, the levels of metabolites are simultaneously disturbed during disease development in vivo, which reflect the disease status; ${ }^{\mathbf{1 6}}$ thus, metabolomics can provide an investigation tool for the physiological or pathophysiological intermediates or end-point metabolites under different conditions. ${ }^{17,18}$ Via metabolomics, we can know what has happened in vivo and provide a comprehensive explanation of functional substances. In this study, we used a UPLC-Q/TOF-MS system, multivariate data analysis, and integrated network analysis tools to explore the potential serum metabolites in $\mathrm{APC}^{\mathrm{min} /+}$ mice and then comprehensively revealed the potential preventive mechanism of mirabilite treatment on the $\mathrm{APC}^{\mathrm{min} /+}$ mice.

\section{Materials and methods}

\subsection{Drugs and chemical reagents}

Mirabilite was provided by TongRentang Pharmacy (Harbin, China). Acetonitrile and methanol were obtained from Thermo 
Fisher Scientific. The ELISA kit was obtained from Nanjing Jian Cheng Bioengineering Institute (Nanjing City, P. R China).

\subsection{Animals and experimental design}

$\mathrm{APC}^{\mathrm{min} /+}$-C57BL/6J $\left(\mathrm{APC}^{\mathrm{min} /+}\right.$ ) mice (N000132) and $\mathrm{C} 57 \mathrm{BL} / 6 \mathrm{~J}$ mice were purchased from Nanjing biomedical research institute of Nanjing University (Nanjing, China). The mice in the mirabilite group were continuously treated with mirabilite at the dose of $0.1 \mathrm{ml} / 10 \mathrm{~g}$ once per day by oral gavage for 10 weeks. Distilled water was administered to the mice in the control group (CON) per day. The experimental procedures were approved by the Animal Care and Ethics Committee at Heilongjiang University of Chinese Medicine, and all experiments were performed in accordance to the declaration of Helsinki.

\subsection{Serum sample collection and preparation}

After the administration of mirabilite for 10 weeks, a total of 15 mice in the experiment were fasted for 12 hours and then sacrificed. Blood samples were obtained from the eyeball. The serum samples were centrifuged at $4000 \mathrm{~g}$ for $15 \mathrm{~min}$ at $4{ }^{\circ} \mathrm{C}$. The supernatant was immediately stored at $-80{ }^{\circ} \mathrm{C}$. Before the UPLC-MS analysis, the serum samples were thawed in an ice water bath. Then, $50 \mu \mathrm{l}$ of serum was added to $200 \mu \mathrm{l}$ of methanol to remove macromolecular proteins. After this, the mixture of thawed serum and methanol was centrifuged at $13000 \mathrm{~g}$ for 15 minutes at $4{ }^{\circ} \mathrm{C}$, and the supernatant was transferred to an HPLC vial and stored at $4{ }^{\circ} \mathrm{C}$.

\subsection{Chromatographic conditions}

An ultra-high-performance liquid chromatography (UPLC) system and the MassLynx ${ }^{\mathrm{TM}}$ software (V4.1 SCN901) were used for the analysis of serum samples. The chromatographic column used herein was the Acquity UPLC HSS T3 Column (100 mm $\times$ 2.1, $1.8 \mu \mathrm{m}$ ). The column temperature was $40{ }^{\circ} \mathrm{C}$. The gradient elution conditions were set as follows: a linear gradient of $2-16 \%$ A (Formic acid : water $=1: 1000$ ), 0-1.5 $\mathrm{min} ; 16-20 \% \mathrm{~A}, 1.5-$ $2.0 \mathrm{~min} ; 20-60 \% \mathrm{~A}, 2.0-4.0 \mathrm{~min} ; 60-65 \% \mathrm{~A}, 4.0-4.5 \mathrm{~min} ; 65-70 \%$ A, 4.5-8.0 min; $70-100 \%$ A, $8.0-10.0 \mathrm{~min} ; 100-100 \%$ A, $10-$ $12 \mathrm{~min} ; 100-2 \% \mathrm{~A}, 12-12.5 \mathrm{~min}$; and then held at $2 \% \mathrm{~A}$ for $2 \mathrm{~min}$ to equilibrate the column; and the flow rate was $0.300 \mathrm{~mL} \mathrm{~min}^{-1}$.

\subsection{Mass spectrometry}

The Waters Acquity Synapt High-Definition MS System and the MassLynx V4.1 workstation were used to obtain the chromatogram (Waters group company, USA). The detailed setups in the positive ion mode and negative ion mode are described as follows: the capillary voltage was set at $30 \mathrm{kV}$ and the cone voltage was set at $30 \mathrm{~V}$; the desolvation gas flow rate was maintained at $600 \mathrm{~L} \mathrm{~h}^{-1}$ with the cone gas flow rate of $50 \mathrm{~L} \mathrm{~h}^{-1}$; the desolvation temperature was $350{ }^{\circ} \mathrm{C}$ and the source temperature was $110{ }^{\circ} \mathrm{C}$. The scan range was between $\mathrm{m} / \mathrm{z} 50$ and 1000. To ensure precise and stable scanning, a lock-mass of leucine enkephalin was used as a lock spray interface at the flow rate of $5 \mu \mathrm{L} \mathrm{min}{ }^{-1}(m / z=556.2771$ in the positive mode and $\mathrm{m} / z$ $=554.2615$ in the negative mode).

\subsection{Multivariate statistical analyses}

The UPLC-MS data was analyzed by the Progenesis QI 1.0 software (Nonlinear Dynamics, 2014, version 1.0) for preconditioning. The procedure consisted of alignment, normalization, and peak picking; multivariate analyses, such as principal component analysis (PCA) and orthogonal partial least squares discriminant analysis (OPLS-DA), were conducted by the EZinfo 2.0 software to generate a multidimensional matrix. The potential information of differential compounds was obtained by screening the results of VIP $>1$ obtained via OPLS-DA; metabolic pathway analysis was executed using the HMDB, ChemSpider, KEGG, and Omicbean databases.

\subsection{Histopathology and enzyme-linked immunosorbent assay}

The mice's abdominal cavity was opened, and the bowel was flushed with normal saline. The intestinal tissues were fixed in $10 \%$ formalin for histopathological analysis. The fixed and stained sections were evaluated by experienced pathology professionals. Images were obtained at the magnification of $100 \times$. The intensity of expression was processed by Image-Pro Plus. The enzyme-linked immunosorbent assay was used to detect the CEA content in serum samples. All operations were performed according to the manufacturer's instructions.

\section{Results}

3.1. Mirabilite prevents colorectal cancer in the $\mathrm{APC}^{\mathrm{min} /+}$ mice

Compared with the case of the mirabilite group, the positive expression of Bax was significantly higher in the model group $(P<$ 0.05). Moreover, the value of Bax/Bcl-2 was lower in the mirabilite group, which suggested that mirabilite had a significant inhibitory effect on cell apoptosis during the developing stages of CRC (Fig. 1A-C). The results of routine examinations showed that mirabilite had positive effects on the prevention and treatment of CRC. CEA, a clinical serum marker, was chosen to investigate the effect of mirabilite on CRC. According to the ELISA detection results, the CEA level was higher in the MOD group than that in the mirabilite group $(P<0.05)$ (Fig. 1D). Furthermore, the histopathological results showed that an obvious variation occurred in the model group (Fig. 1E). The histological analysis results showed that regional intestinal epithelial cells were disorderly arranged; comparatively, the mirabilite group presented an attenuated disorder of these cells (Fig. 1E); via the determination of the apoptotic factors, the role of mirabilite in the prevention of CRC was further explained (Fig. 1F and G).

\subsection{Serum metabolic profile analysis of the $\mathrm{APC}^{\mathrm{min} /+}$ mice}

The metabolic profiles of serum samples on the 70th day were scanned and obtained in both the positive and the negative ion mode (Fig. 2A and B, respectively). The 2D ion intensity map showed approximately 9537 ion peaks in the positive ion mode and 10863 ion peaks in the negative ion mode (Fig. 2C and D, respectively). 

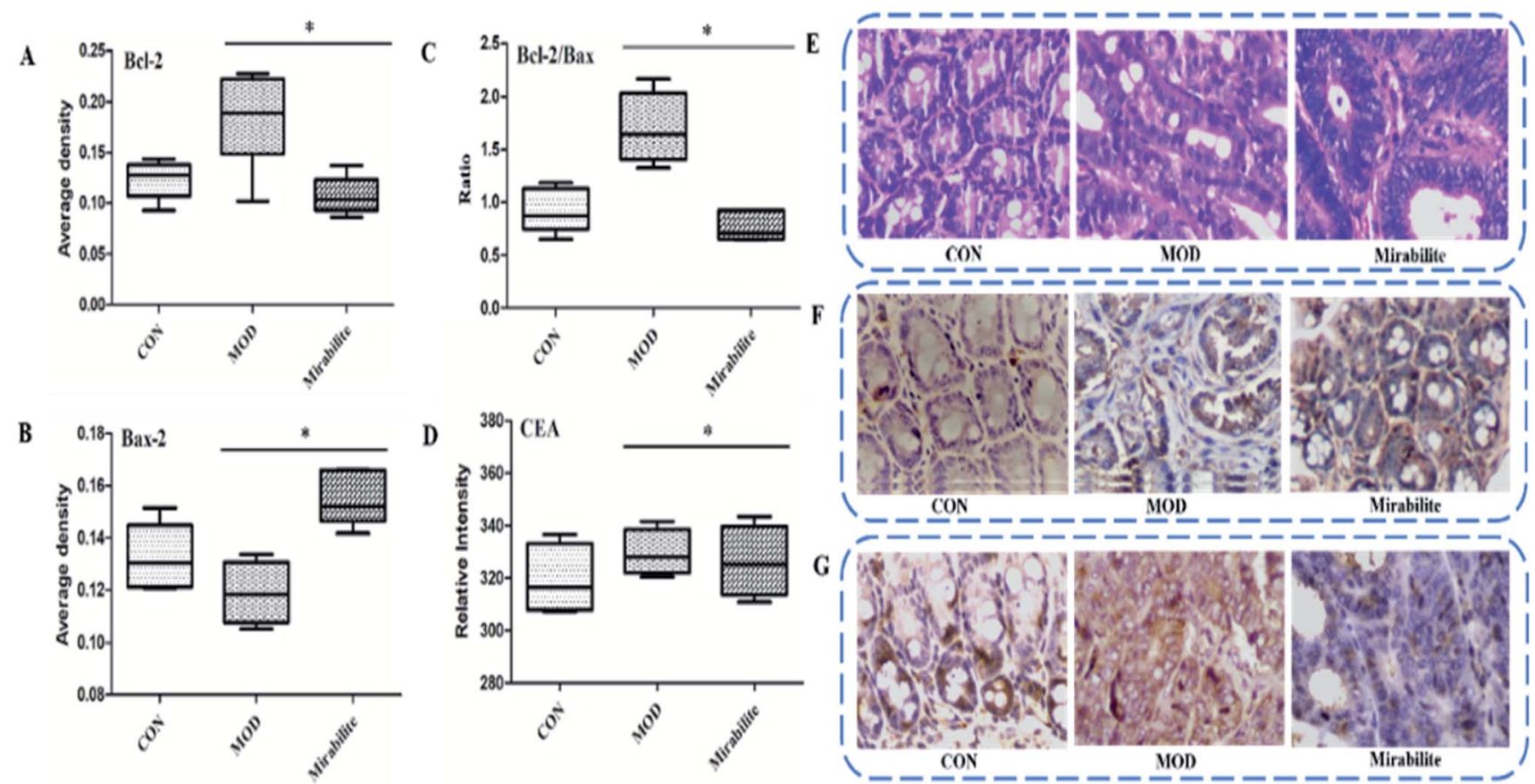

Fig. 1 The apoptotic factors and clinical biochemical indicators. (A-C) show the average density of Bcl-2, the average density of Bax and the ratio of $\mathrm{Bcl}-2$ to Bax in the CON, MOD, and mirabilite groups, respectively. (D) shows the relative intensity of CEA. (E) shows the histopathological results; and $(F)$ and $(G)$ show the immunohistochemistry results of $\mathrm{Bax}$ and $\mathrm{Bcl}-2$, respectively $(*, P<0.05)$.

\subsection{Serum metabolite identification of the $\mathrm{APC}^{\mathrm{min} /+}$ mice} The score-plot showed that the CON and MOD groups had obvious differences in both the positive ion mode and the negative ion mode (Fig. 3A and B, respectively); therefore, a VIP of OPLS-DA was applied to filter the metabolic profile data of the serum samples and discriminate the potential biomarkers of CRC (Fig. 3C).
$\mathbf{A}$

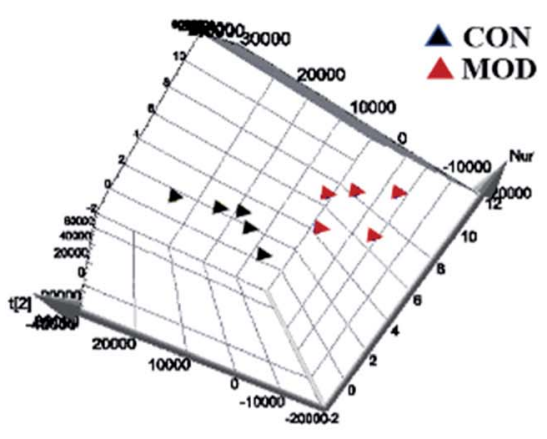

B

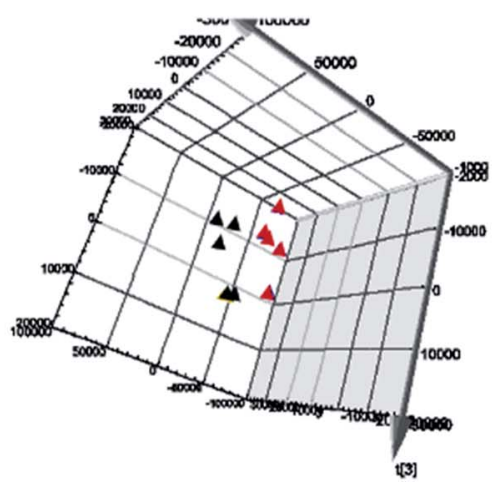

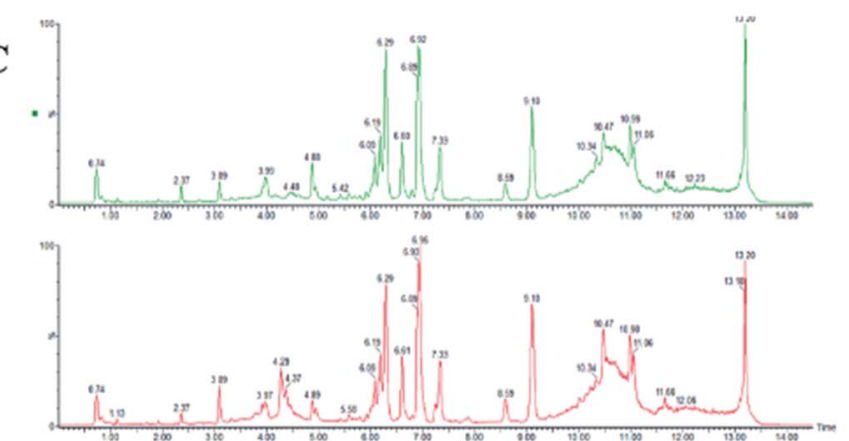

D

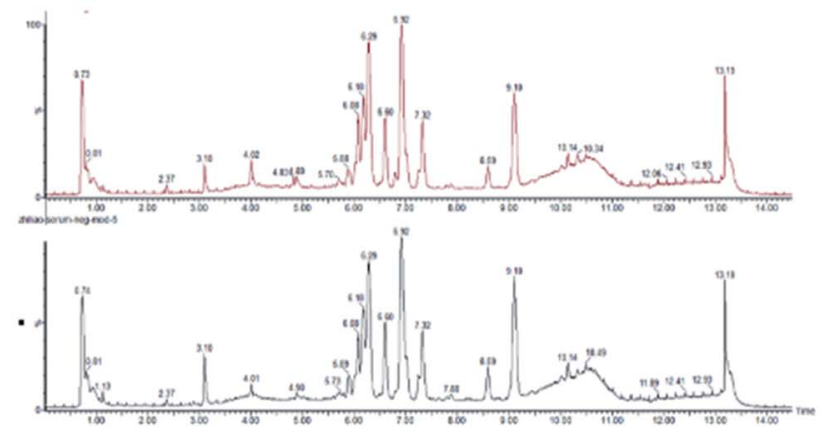

Fig. 2 3D score plots of the PCA model for obtaining metabolic data from serum samples. MOD (red triangles) and CON (black triangles) in the positive mode (A) and negative mode (B). Serum BPI chromatograms of CON and MOD in the positive ion mode (C) and negative ion mode (D). 
Sequentially, the biomarkers were matched according to their detailed fragmentation information with the MassFragment ${ }^{\mathrm{TM}}$ application manager on the basis of accurate MS and MS/MS data. For example, the structure and fragmentation of 5-hydroxyindoleacetic acid are illustrated in Fig. 3D. Finally, a total of 28 biomarkers were identified. Detailed information of compound identification is provided in the attached file (Table S1 $\dagger$ ). A clustering heat map showed the significant changes in the levels of serum metabolites in the CON and APCmin/+ mice (Fig. 4); moreover, a test analysis of relative intensities further demonstrated the changes in the level of each biomarker (Fig. 5).

\subsection{Analysis of the metabolic pathways related to metabolites}

The identified biomarkers were imported into the MetaboAnalyst 4.0 (http://www.metaboanalyst.ca/), Omicbean (http:// www.omicsbean.cn) and KEGG (http://www.kegg.jp/kegg/) databases. The metabolic pathways closely related to these biomarkers were retinol metabolism, glycerophospholipid metabolism, tryptophan metabolism, biosynthesis of unsaturated fatty acids and glycerol-lipid metabolism. The full evaluation is displayed in Fig. 6 and Table S2.†

\subsection{Mirabilite modulates the metabolic profiles}

According to the established preparation method, we obtained the serum samples from the MOD and the mirabilite group on the 70th day to perform a full scan in the positive and negative ion patterns and obtain metabolic information. According to the relative peak intensities, we obtained the scores plot of PCA by EZinfo 2.0 (Fig. 3A and B). From the PCA analysis, it was found that mirabilite could modulate the metabolic profiles of the $\mathrm{APC}^{\mathrm{min} /+}$ mice.

\subsection{Mirabilite regulates the metabolites and their pathways}

Compared with the case of MOD, a total of 18 metabolites were regulated by mirabilite, namely, 5-hydroxyindoleacetic acid, hypoxanthine, retinal, retinal ester, L-acetyl carnitine, 2hydroxybutyric acid, eicosapentaenoic acid, LysoPC(16:1(9Z)), LysoPC(18:0), LysoPC(18:1(9Z)), LysoPC(18:4(6Z,9Z,12Z,15Z)), LysoPC(20:3(5Z,8Z,11Z)), LysoPC(P-18:1(9Z)), LPA(0:0/16:0), LysoPC(15:0), LysoPC(16:0), LysoPC(17:0) and stearic acid, which were associated with high mirabilite efficacy (Fig. 5). Moreover, we found that mirabilite regulated the metabolites in the retinol metabolism, tryptophan metabolism, biosynthesis of unsaturated fatty acids, propanoate metabolism, glycerophospholipid metabolism, fatty acid biosynthesis, and purine metabolism.

\subsection{Network construction}

To further explore the functional alterations, we utilized a comprehensive ingenuity pathway analysis (IPA) system. According to the canonical pathway analysis, 7 related pathways and their predicted networks were identified (Fig. S1A and $\mathrm{B} \dagger$ ). The main pathways included adenosine nucleotide degradation II, purine nucleotide degradation II (aerobic), aryl hydrocarbon receptor signaling, RAR activation, phototransduction pathway,
A



C

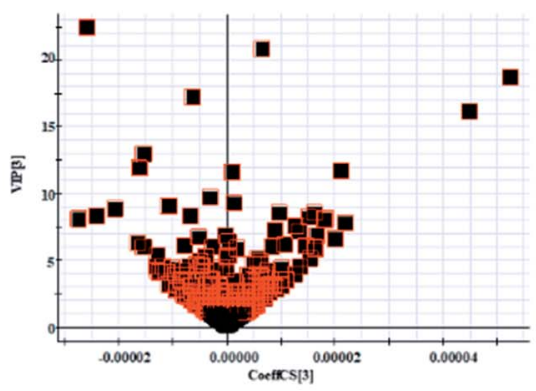

B



D

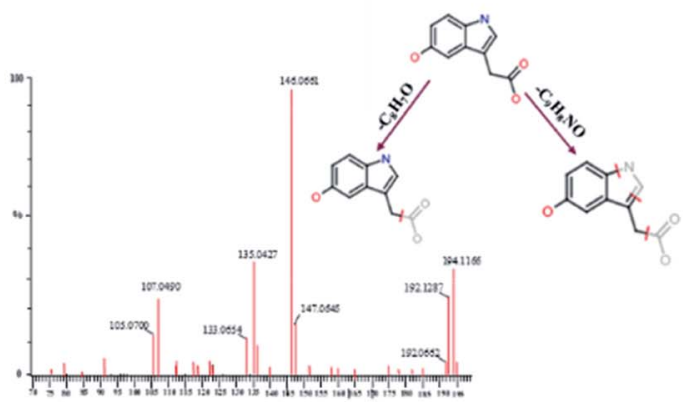

Fig. 3 Score plots of the PCA model for obtaining the metabolomics data from serum samples. MOD (red diamonds) and CON (black diamonds) in the positive mode (A) and negative mode (B). The VIP plot of the selected biomarker (C), VIP > 1 was the criterion to select the potential biomarkers. (D) The chemical structure and mass fragment of 5-hydroxyindoleacetic acid in the negative ion mode. 


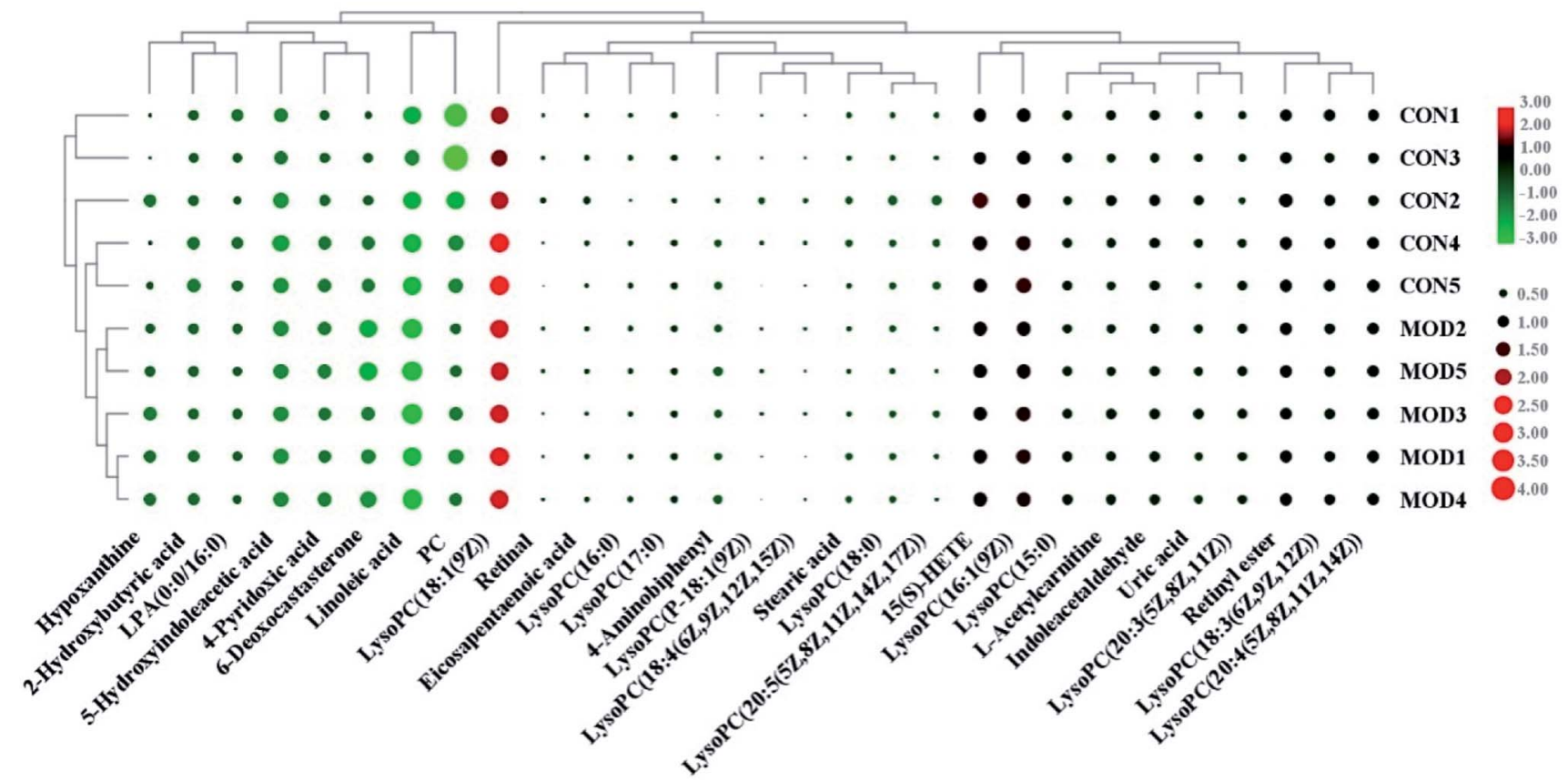

Fig. 4 The clustering heat map of serum metabolites.

urate biosynthesis/inosine $5^{\prime}$-phosphate degradation, and retinol biosynthesis. The predicted networks indicated that 5hydroxyindoleacetic acid, indole acetaldehyde, retinal and retinal ester were the key biomarkers. Interestingly, all these substances were significantly regulated by mirabilite, which could be considered as predictive targets of CRC. Moreover, for the 18 metabolites altered by the treatment of mirabilite, the heatmap showed the clustering of biomarkers between the MOD and mirabilite groups (Fig. 4); the IPA showed that the canonical pathways changed with the treatment, and they were aryl hydrocarbon receptor signaling pathway, RAR activation pathway, phototransduction pathway, retinol biosynthesis, serotonin receptor signaling pathway, the visual cycle, stearate biosynthesis I, and serotonin degradation pathway (Fig. S1C and $\mathrm{D} \dagger$ ). Then, we utilized the MetaboAnalyst 4.0 database to integrate the analysis of differential factors and metabolites and completed the network analysis (Fig. S2 $\dagger$ ); the results showed

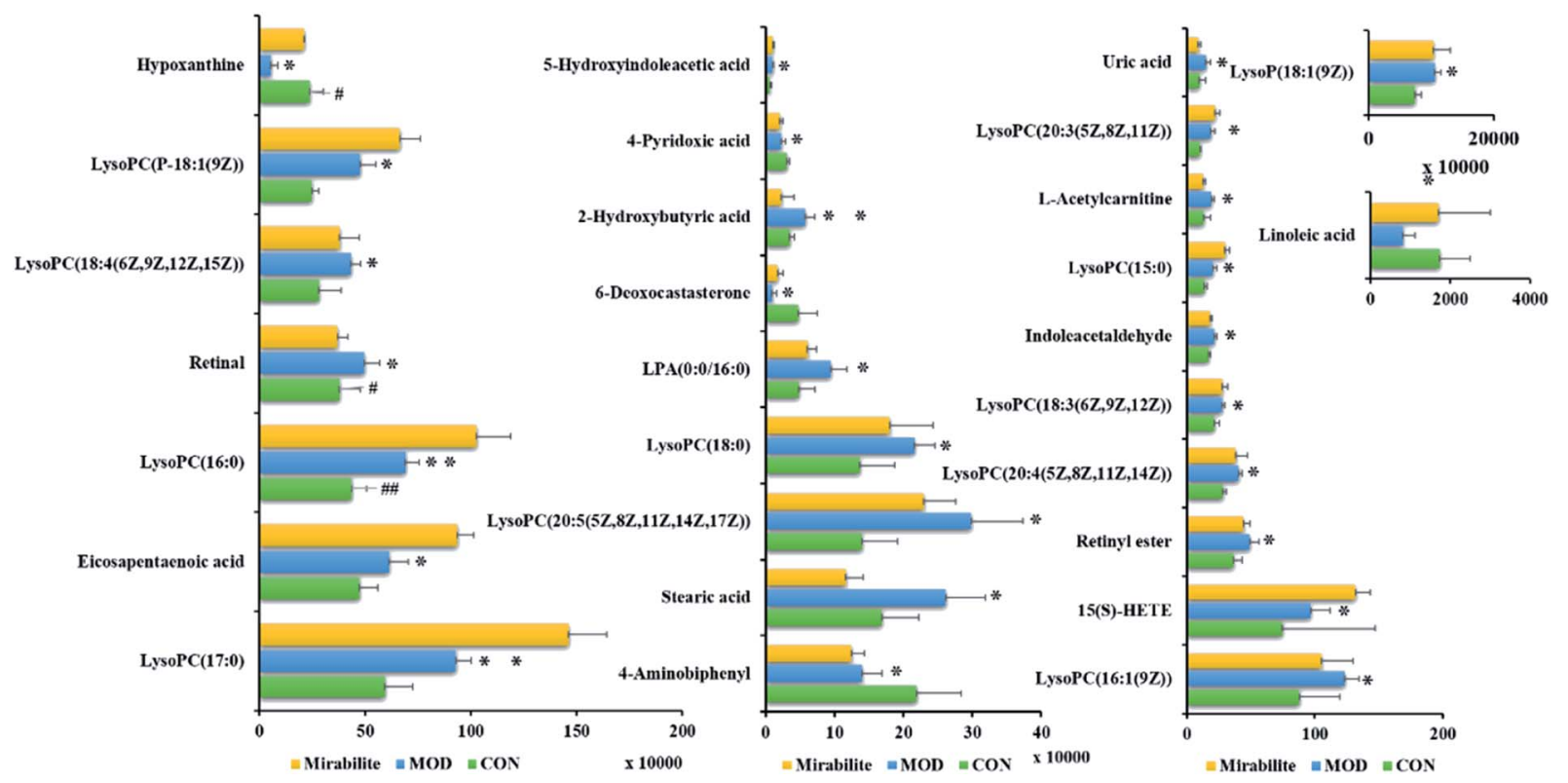

Fig. 5 The expression level of the differential biomarkers in the serum samples. (Compared with CON, "*" was $p<0.05$ and "**" was $p<0.01$; compared with MOD, "\#" was $p<0.05$ and "\#\#*" was $p<0.01$.) 


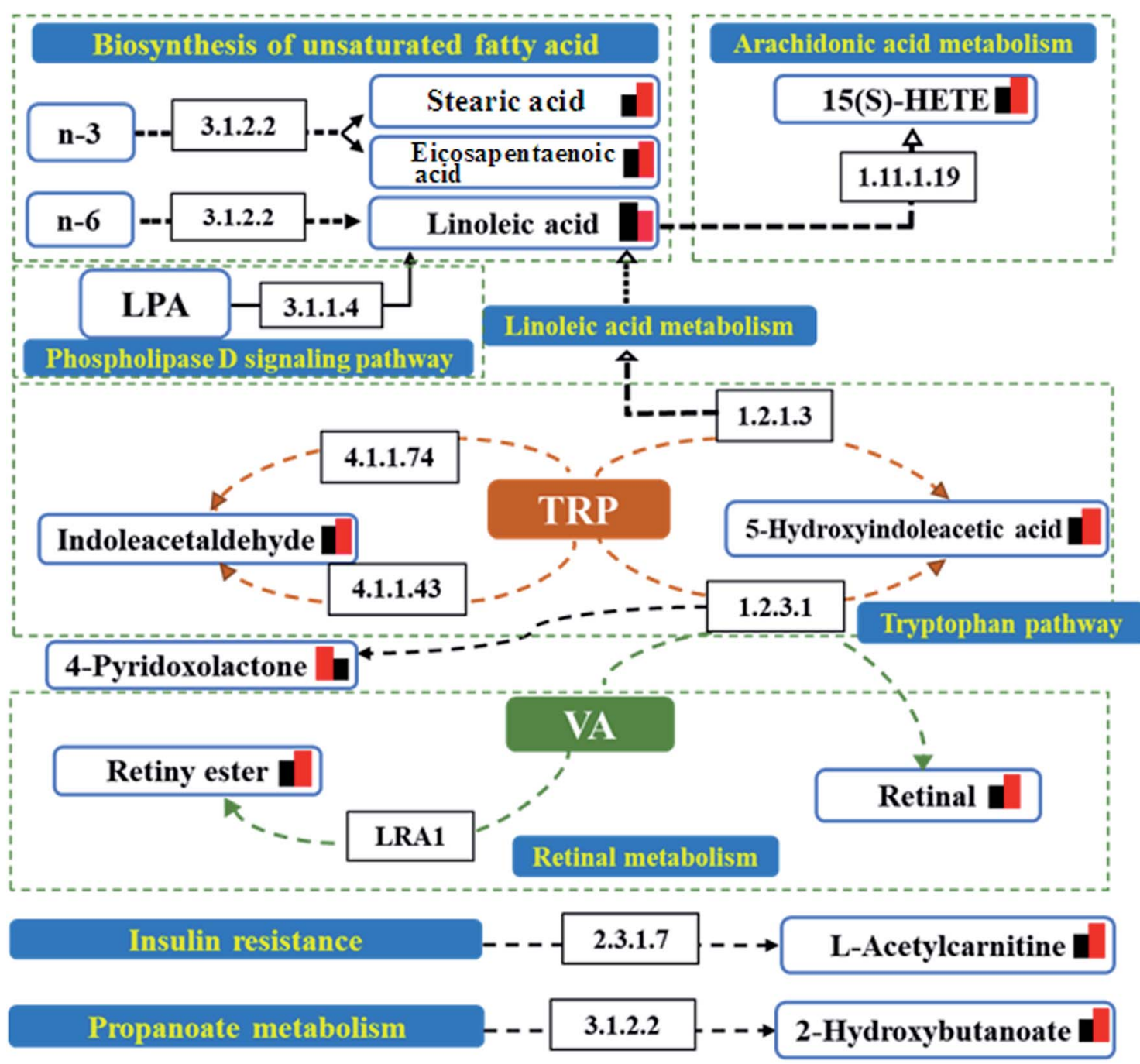

Fig. 6 The metabolic pathway analysis associated with the biomarkers. The red histogram indicates CON, and the black histogram indicates MOD.

that tryptophan metabolism was closely involved in the mirabilite treatment.

\section{Discussion}

CRC begins in the inner parts of the rectum or colon as a tumor and becomes a polyp after abnormal growth; ${ }^{19}$ after this, it is eventually transformed into cancer; the symptoms of CRC are always insidious; therefore, in many patients, CRC is diagnosed in the middle and late stages that have the highest mortality rate. The APCmin/+ mice are the most frequently used intestinal tumor model to study intestinal diseases. ${ }^{20}$ In this study, H\&E staining was performed, and the results showed that mirabilite inhibited the pathogenesis of CRC. Furthermore, the significantly decreased apoptotic factors in the treatment group further indicated that mirabilite had an inhibitory effect on the proliferation of intestinal tumor cells.

We used the metabolomic technologies to explore the key metabolite biomarkers and related metabolic pathways in CRC for deciphering the preventive mechanism of mirabilite on colorectal cancer via the modulation of complex metabolic networks. Among the biomarkers, more than half of the metabolites belonged to the LysoPC class compounds, and the levels of all the LysoPC compounds were increased upon treatment with mirabilite. As is known, lipid metabolism is related to $\mathrm{CRC}^{21}$ An abnormal expression of lipid metabolism suggested that the energy metabolism was disordered, and tumor proliferation was accelerated. ${ }^{22}$ This also reflected the state of intestinal permeability. ${ }^{23}$ After the administration of mirabilite, the levels of some of the lipid metabolites, such as LysoPC(15:0), LysoPC(16:0), LysoPC(16:1(9Z)), returned to normal.

Tryptophan (TRP), an important regulatory factor in the dynamic balance of mucous membrane, has many nutritional and physiological functions. ${ }^{\mathbf{2 4 2 5}}$ It is an important neurotransmitter and signal component involved in the regulation of gastrointestinal motility, visceral sensitivity, and secretion. Moreover, 5-hydroxytryptamine (5-HT) is a relatively less common metabolite of the tryptophan metabolism and mainly 
stored in the small intestine..$^{\mathbf{2 4 , 2 5}}$ The gastrointestinal mucosa is spread throughout the enterochromaffin cells (EC), which generate and store about $90 \%$ of 5 -HT in the intestines. As a very important monoamine excitatory neurotransmitter, 5-HIAA is widely present in the central nervous system and the gastrointestinal tract and participates in gastrointestinal motility. 5HIAA acts as a tumor marker in the study of the metabolic pathway of CRC. The increased intensity of indole acetaldehyde in the model group illustrated that the 5-HT metabolism was enhanced in CRC. The IPA analysis showed the possible target of CRC, and the aryl hydrocarbon receptor signaling pathway was one of the key pathways. Previous studies have shown that ARH affects the normal function of immune cells in the barrier organs. ${ }^{26}$ It has a relationship with the differentiation and/or functions of $\mathrm{T}$ cells, macrophages and dendritic cells. ${ }^{27,28} \mathrm{~A}$ study has displayed that the TRP metabolites are endogenous ligands for AHR, ${ }^{29}$ a key regulator of inflammation and immunity. ${ }^{\mathbf{2 4 0} 30}$ From the serum metabolite analysis results, it was found that both 5-hydroxyindoleacetic acid (5-HIAA) and indole acetaldehyde were involved in the tryptophan metabolism pathway, and their levels were down-regulated by mirabilite. Thus, the ARH signaling pathway was inhibited, and the immune function was recovered in the APCmin/+ mice.

The levels of both hypoxanthine and uric acid were higher in the model group than those in the control group. Hypoxanthine is the precursor of uric acid. Purine metabolism not only participates in gene construction but also promotes cell survival and proliferation. Studies have shown that the purine metabolism contributes to the development of cancer. ${ }^{31}$ The increased level of hypoxanthine suggested that the purine nucleotide degradation was perturbed. Compared to the case of the control group, the level of hypoxanthine was significantly decreased in the mirabilite group. This means that mirabilite can attenuate this disorder to prevent CRC.

\section{Conclusions}

In this study, based on the metabolomics methods, the serum metabolomic profiles were obtained by UPLC-Q/TOF-MS for the analysis of the small molecule metabolites of $\mathrm{APC}^{\mathrm{min} /+}$; the multivariate data analysis showed the variation in the levels of the 28 metabolites that were involved in the tryptophan metabolism, glycerophospholipid metabolism, and biosynthesis of unsaturated fatty acids. Moreover, it was discovered that mirabilite could regulate metabolic disorders and pathways in the APC ${ }^{\mathrm{min} /+}$ mice. Mirabilite mainly regulated tryptophan metabolism and glycerophospholipid metabolism to prevent CRC. This study also shows that high-throughput metabolomics can reveal the perturbed metabolic disorders to determine the action mechanism of drug treatment.

\section{Conflicts of interest}

There are no conflicts to declare.

\section{Acknowledgements}

This work was supported by the grants received from the Key Program of Natural Science Foundation of State (Grant No. 81830110, 8181101160, 81973745, 81903818, 81903847, 81430093, 81302905), National Key Research and Development Program of China (2018YFC1706103), National Key Subject of Drug Innovation (Grant No. 2015ZX09101043-005, 2015ZX09101043-011), TCM State Administration Subject of Public Welfare (Grant No. 2015468004), Natural Science Foundation of Heilongjiang Province (YQ2019H030, LH2019H056, H2016056), University Nursing Program for Young Scholars with Creative Talents in Heilongjiang Province (UNPYSCT2015118, UNPYSCT-2016213, UNPYSCT-2016212), Young Talent Lift Engineering Project of China Association of Traditional Chinese Medicine (QNRC2-B06), and Foundation of Heilongjiang University of Chinese Medicine (2018jc01, 2018bs02, 201809), Heilongjiang Touyan Innovation Team Program.

\section{References}

1 M. Arnold, M. S. Sierra, M. Laversanne, et al. Global patterns and trends in colorectal cancer incidence and mortality, Gut, 2017, 66(4), 683-691.

2 M. J. Schell, M. Yang, J. K. Teer, et al. A multigene mutation classification of 468 colorectal cancers reveals a prognostic role for APC, Nat. Commun., 2016, 7, 11743.

3 J. Rimbert, G. Tachon, A. Junca, et al. Association between clinicopathological characteristics and RAS mutation in colorectal cancer, Mod. Pathol., 2018, 31(3), 517-526.

4 H. Dopeso, P. Rodrigues, J. Bilic, et al. Mechanisms of inactivation of the tumour suppressor gene RHOA in colorectal cancer, Br. J. Cancer, 2018, 118(1), 106-116.

5 D. Shin, J. Lee, J. R. Gong, et al. Percolation transition of cooperative mutational effects in colorectal tumorigenesis, Nat. Commun., 2017, 8(1), 1270.

6 H. Solomon, N. Dinowitz, I. S. Pateras, et al. Mutant p53 gain of function underlies high expression levels of colorectal cancer stem cells markers, Oncogene, 2018 Mar, 37(12), 1669-1684.

7 H. Zhang, A. Zhang, X. Zhou, et al. High-throughput lipidomics reveal mirabilite regulating lipid metabolism as anticancer therapeutics, RSC Adv., 2018, 8(62), 35600-35610.

8 H. Zhang, A. Zhang, J. Miao, et al. Targeting regulation of tryptophan metabolism for colorectal cancer therapy: a systematic review, RSC Adv., 2019, 9(6), 3072-3080.

9 H. Sun, H. L. Zhang, A. H. Zhang, et al. Network pharmacology combined with functional metabolomics discover bile acid metabolism as a promising target for mirabilite against colorectal cancer, $R S C A d v$., 2018, 8(53), 30061-30070.

10 X. J. Wang, J. L. Ren, A. H. Zhang, H. Sun, G. L. Yan, Y. Han and L. Liu, Novel applications of mass spectrometry-based metabolomics in herbal medicines and its active ingredients: current evidence, Mass Spectrom. Rev., 2019, 38(4-5), 380-402. 
11 A. Zhang, H. Sun, G. Yan, et al. Metabolomics in diagnosis and biomarker discovery of colorectal cancer, Cancer Lett., 2014, 345(1), 17-20.

12 H. Sun, A. Zhang, L. Yang, et al. High-throughput chinmedomics strategy for discovering the quality-markers and potential targets for Yinchenhao decoction, Phytomedicine, 2019, 54, 328-338.

13 H. Sun, A. H. Zhang, Q. Song, et al., Functional metabolomics discover pentose and glucuronate interconversion pathways as promising targets for Yang Huang syndrome treatment with Yinchenhao Tang, RSC Adv., 2018, 8, 36831-36839.

14 A. Zhang, H. Sun, Y. Han, et al. Urinary metabolic biomarker and pathway study of hepatitis B virus infected patients based on UPLC-MS system, PLoS One, 2013, 8(5), e64381.

15 Y. Zhang, P. Liu, Y. Li, et al., Exploration of metabolite signatures using high-throughput mass spectrometry coupled with multivariate data analysis, $R S C A d v$., 2017, 7, 6780-6787.

16 Q. Liang, H. Liu, H. Xing, et al. Urinary UPLC-MS metabolomics dissecting the underlying mechanisms of huaxian capsule protects against sepsis, $R S C A d v$., 2016, 6, 40436-40441.

$17 \mathrm{X}$. Wang, A. Zhang, X. Zhou, et al. An integrated chinmedomics strategy for discovery of effective constituents from traditional herbal medicine, Sci. Rep., 2016, 6, 18997.

18 A. Zhang, H. Sun and X. Wang, Emerging role and recent applications of metabolomics biomarkers in obesity disease research, $R S C A d v$. , 2017, 7(25), 14966-14973.

19 J. E. G. Ijspeert, L. Vermeulen, G. A. Meijer, et al. Serrated neoplasia-role in colorectal carcinogenesis and clinical, implications, Nat. Rev. Gastroenterol. Hepatol., 2015 Jul, 12(7), 401-409.

20 Z. Zhong, Y. Zhai, P. Bu, et al. Papilloma-pseudovirus eradicates intestinal tumours and triples the lifespan of Apc(min/+) mice, Nat. Commun., 2017, 8, 15004.
21 A. K. Cotte, V. Aires, M. Fredon, et al. Lysophosphatidylcholine acyltransferase 2-mediated lipid droplet production supports colorectal cancer chemoresistance, Nat. Commun., 2018, 9(1), 322.

22 G. Yan, L. Li, B. Zhu, et al. Lipidome in colorectal cancer, Oncotarget, 2016, 7(22), 33429-33439.

23 R. D. Semba, M. Shardell, I. Trehan, et al. Metabolic alterations in children with environmental enteric dysfunction, Sci. Rep., 2016, 6, 28009.

24 J. Islam, S. Sato, K. Watanabe, et al. Dietary tryptophan alleviates dextran sodium sulfate-induced colitis through aryl hydrocarbon receptor in mice, J. Nutr. Biochem., 2017, 42, 43-50.

25 T. Zelante, R. G. Iannitti, C. Cunha, et al. Tryptophan catabolites from microbiota engage aryl hydrocarbon receptor and balance mucosal reactivity via interleukin-22, Immunity, 2013, 39(2), 372-385.

26 C. Esser and A. Rannug, The aryl hydrocarbon receptor in barrier organ physiology, immunology, and toxicology, Pharmacol. Rev., 2015, 67(2), 259-279.

27 T. Yamada, H. Horimoto, T. Kameyama, et al. Constitutive aryl hydrocarbon receptor signaling constrains type I interferon-mediated antiviral innate defense, Nat. Immunol., 2016, 17(6), 687-694.

28 C. Goudot, A. Coillard, A. C. Villani, et al. Aryl Hydrocarbon Receptor Controls Monocyte Differentiation into Dendritic Cells versus Macrophages, Immunity, 2016, 17(6), 687-694.

29 B. Lamas, M. L. Richard, V. Leducq, et al. CARD9 impacts colitis by altering gut microbiota metabolism of tryptophan into aryl hydrocarbon receptor ligands, Nat. Med., 2016, 22(6), 598-605.

30 J. Islam, S. Sato, K. Watanabe, et al. Dietary tryptophan alleviates dextran sodium sulfate-induced colitis through aryl hydrocarbon receptor in mice, J. Nutr. Biochem., 2017, 42, 43-50.

31 A. M. Pedley and S. J. Benkovic, A New View into the Regulation of Purine Metabolism: The Purinosome, Trends Biochem. Sci., 2017, 42(2), 141-154. 